\title{
FORWARD-BACKWARD RESOLVENT SPLITTING METHODS FOR GENERAL MIXED VARIATIONAL INEQUALITIES
}

\author{
MUHAMMAD ASLAM NOOR, MUZAFFAR AKHTER, \\ and KHALIDA INAYAT NOOR
}

Received 27 October 2002

\begin{abstract}
We use the technique of updating the solution to suggest and analyze a class of new splitting methods for solving general mixed variational inequalities. It is shown that these modified methods converge for pseudomonotone operators, which is a weaker condition than monotonicity. Our methods differ from the known threestep forward-backward splitting of Glowinski, Le Tallec, and M. A. Noor for solving various classes of variational inequalities and complementarity problems. Since general mixed variational inequalities include variational inequalities and complementarity problems as special cases, our results continue to hold for these problems.
\end{abstract}

2000 Mathematics Subject Classification: 49J40, 90C33.

1. Introduction. Variational inequalities theory is a branch of applicable mathematics with a wide range of applications in industrial, physical, regional, social, pure, and applied sciences. Variational inequalities have been extended and generalized in different directions using new and novel techniques. A useful and significant generalization is called the general mixed variational inequality or variational inequality of the second type. In recent years, several numerical methods for solving variational inequalities have been developed. It is a well-known fact that the projection method and its variant forms, including the Wiener-Hopf equations, cannot be extended for mixed variational inequalities involving the nonlinear terms. These facts motivated us to use the technique of the resolvent operators. In this technique, the given operator is decomposed into the sum of two (or more) monotone operators whose resolvents are easier to evaluate than the resolvent of the original operator. Such type of methods is called the operators splitting methods. This can lead to the development of very efficient methods since one can treat each part of the original operator independently. In the context of variational inequalities, Noor $[11,12,16,17]$ has used the resolvent operator technique to suggest and analyze some two-step forward-backward splitting methods. A useful feature of the forward-backward splitting methods for solving variational inequalities is that the resolvent step involves the subdifferential of the proper, convex, 
and lower semicontinuous part only, and other parts facilitate the problem decomposition. If the nonlinear term involving the general mixed variational inequalities is proper, convex, and lower semicontinuous, then it has been shown that the general mixed variational inequalities are equivalent to the fixed-point and resolvent equations. These alternative formulations have been used to develop a number of iterative-type methods for solving mixed variational inequalities. Noor $[11,12,15,16,17]$ used the technique of updating the solution in conjunction with resolvent operator technique to suggest a number of splitting-type algorithms for various classes of variational inequalities. It has been shown that the convergence of such type of splitting and predictorcorrector type algorithms requires the partially relaxed strong monotonicity, which is a weaker condition than cocoercivity. In this note, we suggest and analyze a new class of forward-backward splitting algorithms for a class of general mixed variational inequalities by modifying the associated fixed-point equation. The new splitting methods are self-adaptive type methods involving the line search strategy, where the step size depends upon the resolvent equation, and the searching direction is a combination of the resolvent residue and the modified extraresolvent direction. Our results include the previous results of Noor [11, 12, 15, 16, 17] for solving different classes of variational inequalities as special cases. Our methods are different from those of Glowinski and Le Tallec [4], which they suggested by using the Lagrange multiplier technique. Haubruge et al. [6] have studied the convergence analysis of the three-step schemes of Glowinski and Le Tallec [4] and applied these three-step iterations to obtain new splitting-type algorithms for solving variational inequalities, separable convex programming, and minimization of a sum of convex functions. They have also proved that three-step iterations lead to highly parallelized algorithms under certain conditions. Using essentially the techniques developed in $[4,6]$, one can obtain several new algorithms for solving variational inequalities from our results. Our results can be viewed as novel applications of the technique of updating the solution as well as a refinement and improvement of previously known results.

2. Preliminaries. Let $H$ be a real Hilbert space whose inner product and norm are denoted by $\langle\cdot, \cdot\rangle$ and $\|\cdot\|$, respectively. Let $K$ be a nonempty closed convex set in $H$. Let $\varphi: H \rightarrow R \cup\{+\infty\}$ be a function.

For given nonlinear operators $T, g: H \rightarrow H$, consider the problem of finding $u \in H$ such that

$$
\langle T u, g(v)-g(u)\rangle+\varphi(g(v))-\varphi(g(u)) \geq 0, \quad \forall g(v) \in H
$$

The inequality of type (2.1) is called the general mixed variational inequality or the general variational inequality of the second kind. If the function $\varphi(\cdot)$ is a proper, convex, and lower semicontinuous function, then problem (2.1) is 
equivalent to finding $u \in H$ such that

$$
0 \in T u+\partial \varphi(g(u))
$$

which is known as the problem of finding a zero of the sum of two (maximal) monotone operators and has been studied extensively in recent years.

We remark that if $g \equiv I$, where $I$ is the identity operator, then problem (2.1) is equivalent to finding $u \in H$ such that

$$
\langle T u, v-u\rangle+\varphi(v)-\varphi(u) \geq 0, \quad \forall v \in H,
$$

which is called the mixed variational inequality. It has been shown that a wide class of linear and nonlinear problems arising in finance, economics, circuit and network analysis, elasticity, optimization, and operations research can be studied via the mixed variational inequalities (2.1) and (2.3). For the applications, numerical methods, and formulations, see $[1,2,3,4,5,6,9,10$, $11,12,15,16,20]$.

We note that if $\varphi$ is the indicator function of a closed convex set $K$ in $H$, that is,

$$
\varphi(u) \equiv I_{K}(u)= \begin{cases}0, & \text { if } u \in K, \\ +\infty, & \text { otherwise, }\end{cases}
$$

then problem (2.1) is equivalent to finding $u \in H, g(u) \in K$ such that

$$
\langle T u, g(v)-g(u)\rangle \geq 0 \quad \forall g(v) \in K .
$$

The inequality of type (2.5) is known as the general variational inequality, which was introduced and studied by Noor [7]. It turned out that the oddorder and nonsymmetric free, unilateral, obstacle, and equilibrium problems can be studied by the general variational inequality $(2.5)$, see $[7,8,9,14,18]$.

From now on, we assume that the operator $g$ is onto $K$ and $g^{-1}$ exists unless otherwise specified.

If $K^{*}=\{u \in H:\langle u, v\rangle \geq 0$, for all $v \in K\}$ is a polar cone of a convex cone $K$ in $H$, then problem (2.5) is equivalent to finding $u \in H$ such that

$$
g(u) \in K, \quad T u \in K^{*}, \quad\langle T u, g(u)\rangle=0,
$$

which is known as the general complementarity problem, which was introduced and studied by Noor [7]. We note that if $g(u)=u-m(u)$, where $m$ is a point-to-point mapping, then problem (2.6) is called the quasi-implicit complementarity problem, see the references for the formulation and numerical methods.

For $g \equiv I$, where $I$ is the identity operator, problem (2.5) collapses to finding $u \in K$ such that

$$
\langle T u, v-u\rangle \geq 0, \quad \forall v \in K
$$


which is called the standard variational inequality, introduced and studied by Stampacchia [19]. For the recent state of the art, see the references.

It is clear that problems (2.3), (2.5), (2.6), and (2.7) are special cases of the general mixed variational inequality (2.1). In brief, for a suitable and appropriate choice of the operators $T, g$, and $\varphi$ and the space $H$, one can obtain a wide class of variational inequalities and complementarity problems. This clearly shows that problem (2.1) is a quite general and unifying one. Furthermore, problem (2.1) has important applications in various branches of pure and applied sciences, see the references.

We now recall some well-known concepts and results.

Definition 2.1. For all $u, v, z \in H$, an operator $T: H \rightarrow H$ is said to be

(i) g-monotone if

$$
\langle T u-T v, g(u)-g(v)\rangle \geq 0
$$

(ii) g-pseudomonotone if

$$
\langle T u, g(v)-g(u)\rangle \geq 0 \Longrightarrow\langle T v, g(v)-g(u)\rangle \geq 0
$$

For $g \equiv I$, where $I$ is the identity operator, Definition 2.1 reduces to the classical definition of monotonicity and pseudomonotonicity. It is known that monotonicity implies pseudomonotonicity but the converse is not true, see [2]. Thus we conclude that the concept of pseudomonotonicity is weaker than monotonicity.

DEFINITION 2.2. If $A$ is maximal monotone operator on $H$, then, for a constant $\rho>0$, the resolvent operator associated with $A$ is defined as

$$
J_{A}(u)=(I+\rho A)^{-1}(u), \quad \forall v \in H,
$$

where $I$ is the identity operator. It is well known that the operator $A$ is maximal monotone if and only if the resolvent operator $J_{A}$ is defined everywhere on the space. The operator $J_{A}$ is single valued and nonexpansive.

REMARK 2.3. It is well known that the subdifferential $\partial \varphi$ of a proper, convex, and lower semicontinuous function $\varphi: H \rightarrow R \cup\{\infty\}$ is a maximal monotone operator, so

$$
J_{\varphi}(u)=(I+\partial \varphi)^{-1}(u), \quad \forall u \in H,
$$

is the resolvent operator associated with $\partial \varphi$ and is defined everywhere. 
LEMMA 2.4. For a given $z \in H, u \in H$ satisfies

$$
\langle u-z, v-u\rangle+\rho \varphi(v)-\rho \varphi(u) \geq 0, \quad \forall v \in H,
$$

if and only if

$$
u=J_{\varphi} z
$$

where $J_{\varphi}$ is the resolvent operator.

We remark that if the proper, convex, and lower semicontinuous function $\varphi$ is an indicator function of closed convex set $K$ in $H$, then $J_{\varphi} \equiv P_{K}$, the projection of $H$ onto $K$. In this case, Lemma 2.4 is equivalent to the projection lemma, see [1].

3. Main results. In this section, we use the resolvent operator technique to suggest a modified resolvent method for solving general mixed variational inequalities of type (2.1). For this purpose, we need the following result, which can be proved using Lemma 2.4 .

LEMMA 3.1. The general mixed variational inequality (2.1) has a solution $u \in H$ if and only if $u \in H$ satisfies

$$
g(u)=J_{\varphi}[g(u)-\rho T u]
$$

where $J_{\varphi}=(I+\rho \partial \varphi)^{-1}$ is the resolvent operator.

Lemma 3.1 implies that problems (2.1) and (3.1) are equivalent. This alternative equivalent formulation has played an important part in suggesting several iterative methods for solving general mixed variational inequalities and related problems, see $[4,5,6,11,12,14,15,16,17]$.

In recent years, the technique of updating the solution has been used to suggest and analyze a number of iterative methods for solving variational inequalities. The main idea in this technique is to modify the resolvent method by performing an additional step forward and a resolvent at each iteration. Using the technique of updating the solution, one can rewrite (3.1) in the form

$$
\begin{aligned}
g(u) & =J_{\varphi}[g(w)-\rho T w], \\
g(w) & =J_{\varphi}[g(y)-\rho T y], \\
g(y) & =J_{\varphi}[g(u)-\rho T u] .
\end{aligned}
$$

Invoking Lemma 3.1, one can easily show that $u \in H$ is a solution of (2.1) if and only if $u \in H$ is a zero of the equation

$$
g(u)-J_{\varphi}[g(w)-\rho T w]=0 .
$$


We now define the resolvent residue vector by

$$
R(u)=g(u)-J_{\varphi}[g(y)-\rho T y] \equiv g(u)-g(w),
$$

where $g(y)$ is defined by (3.4) and $g(w)$ by (3.3).

From Lemma 3.1, it follows that $u \in H$ is a solution of (2.1) if and only if $u \in H$ is a zero of the equation

$$
R(u)=0
$$

The above fixed-point formulation is used to suggest and analyze the following iterative methods for solving general mixed variational inequalities (2.1).

Algorithm 3.2. For a given $u_{0} \in H$, compute the approximate solution $u_{n+1}$ by the iterative schemes

$$
\begin{aligned}
g\left(y_{n}\right) & =J_{\varphi}\left[g\left(u_{n}\right)-\rho T u_{n}\right], \\
g\left(w_{n}\right) & =J_{\varphi}\left[g\left(y_{n}\right)-\rho T y_{n}\right], \\
g\left(u_{n+1}\right) & =J_{\varphi}\left[g\left(w_{n}\right)-\rho T w_{n}\right], \quad n=0,1,2, \ldots,
\end{aligned}
$$

which is known as the predictor-corrector method, see Noor [16].

Algorithm 3.3. For a given $u_{0} \in H$, compute the approximate solution $u_{n+1}$ by the iterative scheme

$$
g\left(u_{n+1}\right)=J_{\varphi}\left[I-\rho T g^{-1}\right] J_{\varphi}\left[I-\rho T g^{-1}\right] J_{\varphi}\left[I-\rho T g^{-1}\right] g\left(u_{n}\right), \quad n=0,1,2, \ldots,
$$

which is known as the three-step forward-backward splitting algorithm. Note that the order of $T$ and $J_{\varphi}$ has not been changed. This method is compatible with the three-step forward-backward splitting algorithm of Glowinski and Le Tallec [4]. For the convergence analysis of Algorithm 3.3, see Noor [16] and Haubruge et al. [6].

By rearranging the terms, one can use the fixed-point formulation (3.2), (3.3), and (3.4) to suggest and analyze the following method for solving the general mixed variational inequalities of type (2.1).

Algorithm 3.4. For a given $u_{0} \in H$, compute $u_{n+1}$ by the iterative scheme

$$
\begin{gathered}
g\left(u_{n+1}\right)=\left(I+\rho T g^{-1}\right)^{-1}\left\{J_{\varphi}\left[I-\rho T g^{-1}\right] J_{\varphi}\left[I-\rho T g^{-1}\right] J_{\varphi}\left[I-\rho T g^{-1}\right]\right. \\
\left.+\rho T g^{-1}\right\} g\left(u_{n}\right), \quad n=0,1,2, \ldots,
\end{gathered}
$$

which is again a three-step forward-backward splitting-type method and can be considered as a generalization of an algorithm of Tseng [20] and Noor [16]. Noor [13] has studied the convergence of Algorithms 3.2, 3.3, and 3.4 for the 
partially relaxed strongly monotone operator, which is a weaker condition than cocoercivity.

In this note, we suggest another method involving the line search strategy, which includes these splitting-type methods as special cases.

For a given positive constant $\alpha$, we rewrite (3.1), using (3.2), (3.3), and (3.4), in the following form:

$$
\begin{aligned}
g(u) & =J_{\varphi}[g(u)-\alpha\{g(u)-g(w)+\rho T w\}] \\
& =J_{\varphi}[g(u)-\alpha\{R(u)+\rho T w\}] \\
& =J_{\varphi}[g(u)-\alpha d(u)],
\end{aligned}
$$

where

$$
d(u)=R(u)+\rho T w \equiv R(u)+\rho T g^{-1} J_{\varphi}[g(y)-\rho T y]
$$

This fixed-point formulation enables us to suggest the following iterative method for general mixed variational inequalities of type (2.1).

Algorithm 3.5. For a given $u_{0} \in H$, compute the approximate solution $u_{n+1}$ by the following iterative schemes.

\section{PREDictor STEP}

$$
\begin{aligned}
& g\left(y_{n}\right)=J_{\varphi}\left[g\left(u_{n}\right)-\rho_{n} T u_{n}\right], \\
& g\left(w_{n}\right)=J_{\varphi}\left[g\left(y_{n}\right)-\rho_{n} T y_{n}\right],
\end{aligned}
$$

where $\rho_{n}$ satisfies

$$
\rho_{n}\left\langle T u_{n}-T w_{n}, R\left(u_{n}\right)\right\rangle \leq \sigma\left\|R\left(u_{n}\right)\right\|^{2}, \quad \sigma \in(0,1) .
$$

\section{CORRECTOR STEP}

$$
g\left(u_{n+1}\right)=J_{\varphi}\left[g\left(u_{n}\right)-\alpha_{n} d\left(u_{n}\right)\right], \quad n=0,1,2, \ldots,
$$

where

$$
\begin{aligned}
d\left(u_{n}\right) & =R\left(u_{n}\right)+\rho_{n} T w_{n} \\
& =R\left(u_{n}\right)+\rho_{n} T g^{-1} J_{\varphi}\left[g\left(y_{n}\right)-\rho_{n} T y_{n}\right], \\
\alpha_{n} & =\frac{\left\langle R\left(u_{n}\right), D\left(u_{n}\right)\right\rangle}{\left\|d\left(u_{n}\right)\right\|^{2}}, \\
D\left(u_{n}\right) & =R\left(u_{n}\right)-\rho_{n} T u_{n}+\rho_{n} T w_{n} \\
& =R\left(u_{n}\right)-\rho_{n} T u_{n}+\rho_{n} T g^{-1} J_{\varphi}\left[g\left(y_{n}\right)-\rho_{n} T y_{n}\right],
\end{aligned}
$$

where $\alpha_{n}$ is the corrector step size. 
If the proper, convex, and lower semicontinuous function $\varphi$ is an indicator function of a closed convex set $K$ in $H$, then $J_{\varphi} \equiv P_{K}$, the projection of $H$ onto $K$, and, consequently, Algorithm 3.5 collapses to the following algorithm.

Algorithm 3.6. For a given $u_{0} \in H$, compute the approximate solution $u_{n+1}$ by the following iterative schemes.

PREDICTOR STEP

$$
\begin{aligned}
& g\left(y_{n}\right)=P_{K}\left[g\left(u_{n}\right)-\rho_{n} T u_{n}\right], \\
& g\left(w_{n}\right)=P_{K}\left[g\left(y_{n}\right)-\rho_{n} T y_{n}\right],
\end{aligned}
$$

where $\rho_{n}$ satisfies

$$
\rho_{n}\left\langle T u_{n}-T w_{n}, R\left(u_{n}\right)\right\rangle \leq \sigma\left\|R\left(u_{n}\right)\right\|^{2}, \quad \sigma \in(0,1)
$$

\section{CORRECTOR STEP}

$$
g\left(u_{n+1}\right)=P_{K}\left[g\left(u_{n}\right)-\alpha_{n} d_{1}\left(u_{n}\right)\right], \quad n=0,1,2, \ldots,
$$

where

$$
\begin{aligned}
d_{1}\left(u_{n}\right) & =R\left(u_{n}\right)+\rho_{n} T w_{n}, \\
\alpha_{n} & =\frac{\left\langle R\left(u_{n}\right), D_{1}\left(u_{n}\right)\right\rangle}{\left\|d_{1}\left(u_{n}\right)\right\|^{2}}, \\
D_{1}\left(u_{n}\right) & =R\left(u_{n}\right)-\rho_{n} T u_{n}+\rho_{n} T w_{n} .
\end{aligned}
$$

Algorithm 3.6 appears to be a new one even for general variational inequality (2.5). Note that, for $\alpha_{n}=1$, Algorithm 3.5 is exactly Algorithm 3.2, which is mainly due to Noor [16]. For $g \equiv I$, where $I$ is the identity operator, we obtain new improved versions of algorithms for variational inequalities and related optimization problems. This clearly shows that Algorithm 3.5 is a unifying one and includes several known and new algorithms as special cases.

For the convergence analysis of Algorithm 3.5, we need the following results.

LEMMA 3.7. If $\bar{u} \in H$ is a solution of (2.1) and $T$ is $g$-pseudomonotone, then

$$
\langle g(u)-g(\bar{u}), d(u)\rangle \geq(1-\sigma)\|R(u)\|^{2}, \quad \forall u \in H
$$

Proof. Let $\bar{u} \in H$ be a solution of (2.1). Then

$$
\langle T \bar{u}, g(v)-g(\bar{u})\rangle+\varphi(g(v))-\varphi g(\bar{u}) \geq 0, \quad \forall v \in H,
$$


which implies

$$
\langle T v, g(v)-g(\bar{u})\rangle+\varphi(g(v))-\varphi(g(\bar{u})) \geq 0
$$

since $T$ is $g$-pseudomonotone.

Taking $g(v)=J_{\varphi}[g(y)-\rho T y]=g(w)$ in (3.25), we have

$$
\langle T w, g(w)-g(\bar{u})\rangle+\varphi(g(w))-\varphi(g(\bar{u})) \geq 0,
$$

from which we have

$$
\langle g(u)-g(\bar{u}), \rho T w\rangle \geq \rho\langle R(u), T w\rangle+\rho \varphi(g(\bar{u}))-\varphi(g(w)) .
$$

Setting $u=g(w), z=g(u)-\rho T u$, and $v=g(\bar{u})$ in (2.12), we have

$$
\langle g(w)-g(u)+\rho T u, g(\bar{u})-g(w)\rangle+\rho \varphi(g(\bar{u}))-\rho \varphi(g(w)) \geq 0,
$$

from which we obtain

$$
\begin{aligned}
\langle g(u)-g(\bar{u}), R(u)\rangle \geq & \langle R(u), R(u)-\rho T u\rangle-\rho \varphi(g(\bar{u}))+\rho \varphi(g(w)) \\
& +\rho\langle T u, g(u)-g(\bar{u})\rangle \\
\geq & \langle R(u), R(u)-\rho T u\rangle-\rho \varphi(g(\bar{u}))+\rho \varphi(g(w)),
\end{aligned}
$$

where we have used the fact that the operator $T$ is pseudomonotone.

Adding (3.27) and (3.29), we have

$$
\begin{aligned}
\langle g(u)-g(\bar{u}), R(u)+\rho T w\rangle & =\langle g(u)-g(\bar{u}), d(u)\rangle \\
& \geq\langle R(u), D(u)\rangle \\
& =\langle R(u), R(u)-\rho T u+\rho T w\rangle \\
& \geq\|R(u)\|^{2}-\rho\langle R(u), T u-T w\rangle \\
& \geq(1-\sigma)\|R(u)\|^{2} \quad \text { using (3.14), }
\end{aligned}
$$

which is the required result.

LEMMA 3.8. Let $\bar{u} \in H$ be a solution of (2.1) and let $u_{n+1}$ be the approximate solution obtained from Algorithm 3.5. If $T$ is $g$-pseudomonotone, then

$$
\left\|g\left(u_{n+1}\right)-g(\bar{u})\right\|^{2} \leq\left\|g\left(u_{n}\right)-g(\bar{u})\right\|^{2}-\frac{(1-\sigma)^{2}\left\|R\left(u_{n}\right)\right\|^{4}}{\left\|d\left(u_{n}\right)\right\|^{2}} .
$$


Proof. From (3.15), (3.17), (3.23), and the second line of (3.30), we have

$$
\begin{aligned}
\left\|g\left(u_{n+1}\right)-g(\bar{u})\right\|^{2} \leq & \left\|g\left(u_{n}\right)-g(\bar{u})-\alpha_{n} d\left(u_{n}\right)\right\|^{2} \\
\leq & \left\|g\left(u_{n}\right)-g(\bar{u})\right\|^{2}-2 \alpha_{n}\left\langle g\left(u_{n}\right)-g(\bar{u}), d\left(u_{n}\right)\right\rangle \\
& +\alpha_{n}^{2}\left\|d\left(u_{n}\right)\right\|^{2} \\
\leq & \left\|g\left(u_{n}\right)-g(\bar{u})\right\|^{2}-\alpha_{n}\left\langle R\left(u_{n}\right), D\left(u_{n}\right)\right\rangle \\
\leq & \left\|g\left(u_{n}\right)-g(\bar{u})\right\|^{2}-\alpha_{n}(1-\sigma)\left\|R\left(u_{n}\right)\right\|^{2} \\
\leq & \left\|g\left(u_{n}\right)-g(\bar{u})\right\|^{2}-\frac{(1-\sigma)^{2}\left\|R\left(u_{n}\right)\right\|^{4}}{\left\|d\left(u_{n}\right)\right\|^{2}},
\end{aligned}
$$

which is the required result.

THEOREM 3.9. Let $g: H \rightarrow H$ be invertible and let $H$ be a finite-dimensional space. If $u_{n+1}$ is the approximate solution obtained from Algorithm 3.5 and $\bar{u} \in H$ is a solution of (2.1), then $\lim _{n \rightarrow \infty} u_{n}=\bar{u}$.

Proof. Let $\bar{u} \in H$ be a solution of (2.1). From (3.31), it follows that the sequence $\left\{\left\|g(\bar{u})-g\left(u_{n}\right)\right\|\right\}$ is nonincreasing and, consequently, $\left\{g\left(u_{n}\right)\right\}$ is bounded. Under the assumptions of $g$, it follows that the sequence $\left\{u_{n}\right\}$ is also bounded. Furthermore, we have

$$
\sum_{n=0}^{\infty} \frac{(1-\sigma)^{2}\left\|R\left(u_{n}\right)\right\|^{4}}{\left\|d\left(u_{n}\right)\right\|^{2}} \leq\left\|g\left(u_{0}\right)-g(\bar{u})\right\|^{2},
$$

which implies that

$$
\lim _{n \rightarrow \infty} R\left(u_{n}\right)=0
$$

Let $\hat{u}$ be the cluster point of $\left\{u_{n}\right\}$, and the subsequence $\left\{u_{n_{j}}\right\}$ of the sequence $\left\{u_{n}\right\}$ converges to $\hat{u} \in H$. Since $R(u)$ is continuous, so

$$
R(\hat{u})=\lim _{j \rightarrow \infty} R\left(u_{n_{j}}\right)=0
$$

which implies that $\hat{u}$ solves the general mixed variational inequality (2.1) by invoking Lemma 3.1. From (3.31) and (3.34), it follows that

$$
\left\|g\left(u_{n+1}\right)-g(\bar{u})\right\|^{2} \leq\left\|g\left(u_{n}\right)-g(\bar{u})\right\|^{2}
$$

Thus it follows from the above inequality that the sequence $\left\{u_{n}\right\}$ has exactly one cluster point $\hat{u}$ and

$$
\lim _{n \rightarrow \infty} g\left(u_{n}\right)=g(\hat{u}) .
$$


Since $g$ is invertible, so

$$
\lim _{n \rightarrow \infty}\left(u_{n}\right)=\hat{u}
$$

which is the required result.

\section{REFERENCES}

[1] C. Baiocchi and A. Capelo, Variational and Quasivariational Inequalities, A WileyInterscience Publication, John Wiley \& Sons, New York, 1984.

[2] F. Giannessi and A. Maugeri (eds.), Variational Inequalities and Network Equilibrium Problems, Plenum Press, New York, 1995.

[3] F. Giannessi, A. Maugeri, and P. M. Pardalos (eds.), Equilibrium Problems: Nonsmooth Optimization and Variational Inequality Models, Kluwer Academic Publishers, Dordrecht, 2001.

[4] R. Glowinski and P. Le Tallec, Augmented Lagrangian and Operator-Splitting Methods in Nonlinear Mechanics, SIAM Studies in Applied Mathematics, vol. 9, SIAM, Pennsylvania, 1989.

[5] R. Glowinski, J. L. Lions, and R. Trémolières, Numerical Analysis of Variational Inequalities, Studies in Mathematics and Its Applications, vol. 8, NorthHolland Publishing, Amsterdam, 1981.

[6] S. Haubruge, V. H. Nguyen, and J. Strodiot, Convergence analysis and applications of the Glowinski-Le Tallec splitting method for finding a zero of the sum of two maximal monotone operators, J. Optim. Theory Appl. 97 (1998), no. 3, 645-673.

[7] M. A. Noor, General variational inequalities, Appl. Math. Lett. 1 (1988), no. 2, 119-122.

[8] _ Wiener-Hopf equations and variational inequalities, J. Optim. Theory Appl. 79 (1993), no. 1, 197-206.

[9] _ Some recent advances in variational inequalities. I. Basic concepts, New Zealand J. Math. 26 (1997), no. 1, 53-80.

[10] _ Some recent advances in variational inequalities. II. Other concepts, New Zealand J. Math. 26 (1997), no. 2, 229-255.

[11] _ A modified extragradient method for general monotone variational inequalities, Comput. Math. Appl. 38 (1999), no. 1, 19-24.

[12] _ Some algorithms for general monotone mixed variational inequalities, Math. Comput. Modelling 29 (1999), no. 7, 1-9.

[13] _ A class of new iterative methods for general mixed variational inequalities, Math. Comput. Modelling 31 (2000), no. 13, 11-19.

[14]_, New approximation schemes for general variational inequalities, J. Math. Anal. Appl. 251 (2000), no. 1, 217-229.

[15] _ Projection-splitting algorithms for monotone variational inequalities, Comput. Math. Appl. 39 (2000), no. 9-10, 73-79.

[16]__ Splitting algorithms for general pseudomonotone mixed variational inequalities, J. Global Optim. 18 (2000), no. 1, 75-89.

[17]__ Splitting methods for pseudomonotone mixed variational inequalities, J. Math. Anal. Appl. 246 (2000), no. 1, 174-188.

[18] M. A. Noor, K. I. Noor, and Th. M. Rassias, Some aspects of variational inequalities, J. Comput. Appl. Math. 47 (1993), no. 3, 285-312.

[19] G. Stampacchia, Formes bilinéaires coercitives sur les ensembles convexes, C. R. Acad. Sci. Paris 258 (1964), 4413-4416 (French). 
[20] P. Tseng, A modified forward-backward splitting method for maximal monotone mappings, SIAM J. Control Optim. 38 (2000), no. 12, 431-446.

Muhammad Aslam Noor: Etisalat College of Engineering, P.O. Box 980, Sharjah, United Arab Emirates

E-mail address: noor@ece.ac.ae

Muzaffar Akhter: Etisalat College of Engineering, P.O. Box 980, Sharjah, United Arab Emirates

E-mail address: makhter@ece.ac.ae

Khalida Inayat Noor: Department of Mathematics and Computer Science, College of Science, United Arab Emirates University, P.O. Box 17551, Al Ain, United Arab Emirates

E-mail address: kha7idan@uaeu. ac.ae 


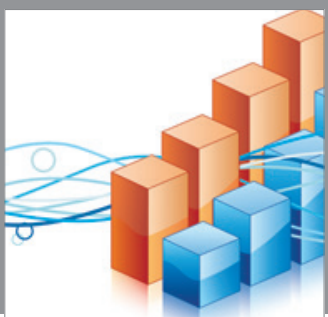

Advances in

Operations Research

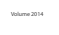

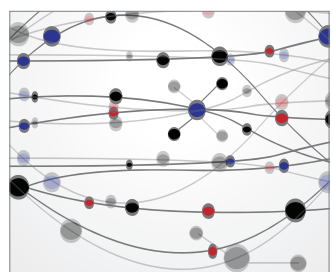

\section{The Scientific} World Journal
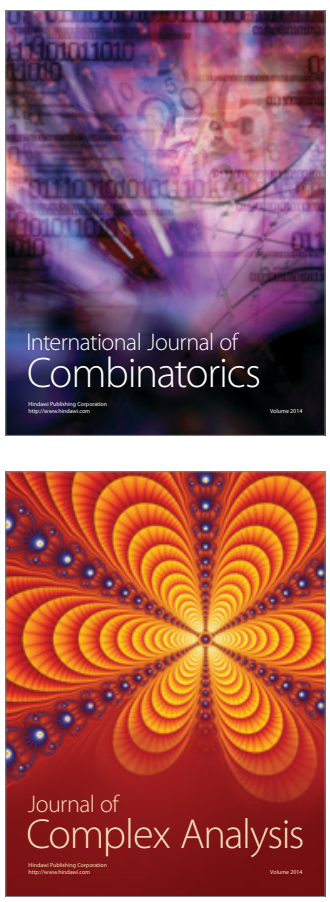

International Journal of

Mathematics and

Mathematical

Sciences
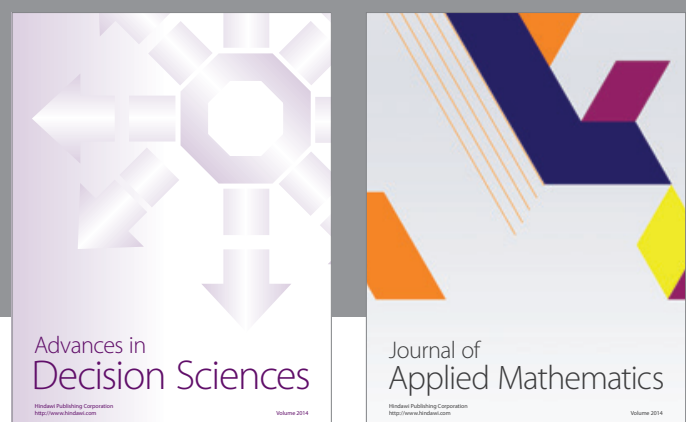

Journal of

Applied Mathematics
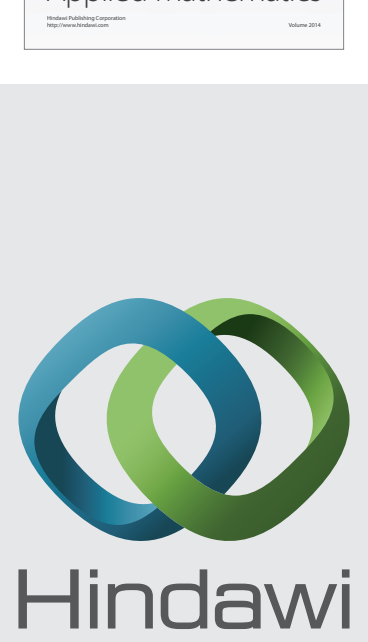

Submit your manuscripts at http://www.hindawi.com
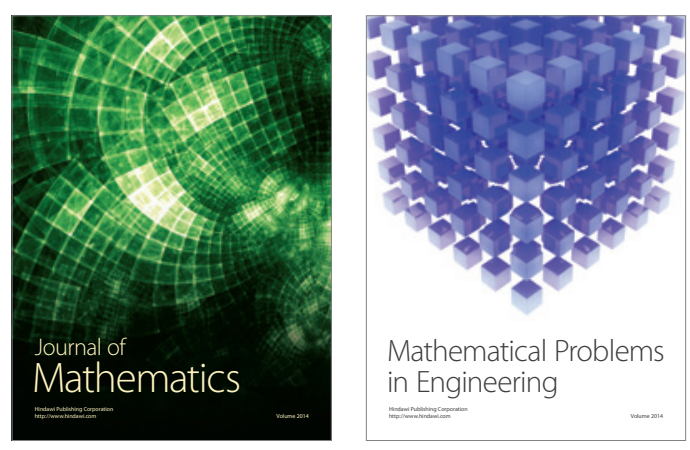

Mathematical Problems in Engineering
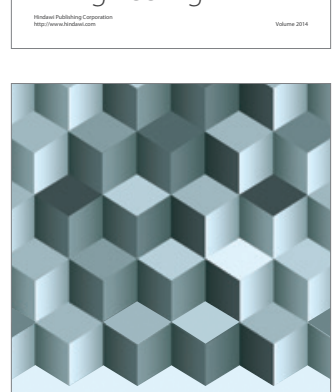

Journal of

Function Spaces
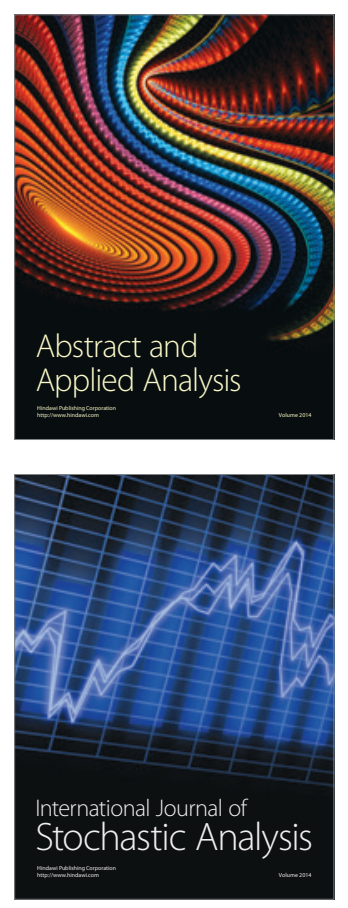

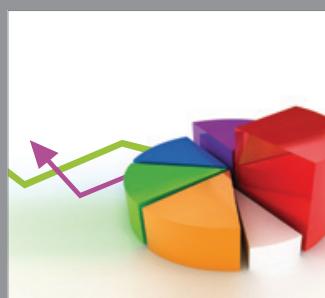

ournal of

Probability and Statistics

Promensencen
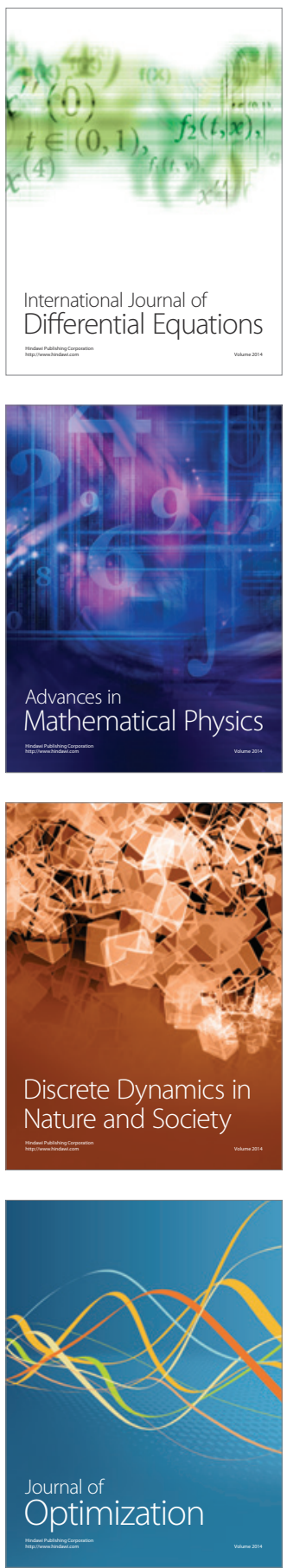